\title{
Character of tightly bound excitons in small argon clusters: Insights from size-dependent energy shifts
}

\author{
T. Laarmann, ${ }^{1, *}$ K. von Haeften, ${ }^{1, \dagger}$ A. Kanaev, ${ }^{2}$ H. Wabnitz, ${ }^{1}$ and T. Möller ${ }^{1}$ \\ ${ }^{1}$ Hamburger Synchrotronstrahlungslabor HASYLAB at Deutsches Elektronen Synchrotron DESY, Notkestr. 85, 22607 Hamburg, Germany \\ ${ }^{2}$ Laboratoire d'Ingénierie des Matériaux et des Hautes Pressions, C.N.R.S. Institut Galilée, Université Paris-Nord, \\ 93430 Villetaneuse, France
}

(Received 21 May 2002; revised manuscript received 03 September 2002; published 15 November 2002)

\begin{abstract}
The structure of the first electronically excited states of small $\operatorname{Ar}_{\bar{M}}$ clusters $(\bar{M} \leqslant 100)$ embedded in large $\mathrm{Ne}_{\bar{N}}(\bar{N}=7500)$ clusters is investigated using fluorescence excitation spectroscopy. In the energy range of the characteristic absorption of Ar clusters (11.5-12.9 eV), surface excitons of Ar clusters embedded in $\mathrm{Ne}$ disappear, while additional absorption bands appear. They are assigned to excitons at the interface between the Ar cluster and the Ne host cluster. The observed energy shift of all absorption bands is proportional to the logarithm of the cluster radius. This can be understood in the Frenkel-exciton model, taking the resonant excitation transfer into account.
\end{abstract}

DOI: 10.1103/PhysRevB.66.205407

PACS number(s): 36.40.Mr, 61.72.Ww, 71.35.Aa, 78.40.- $-\mathrm{q}$

\section{INTRODUCTION}

One important aspect of cluster science is the study of electronic structure size dependence and its convergence toward that of the bulk. In this context rare gas clusters play an important role as model systems for insulators. Recent studies of the electronic structure of $\mathrm{Ar}, \mathrm{Kr}$, and Xe clusters have shown that the weakly bound electronic excitations can be interpreted as Wannier excitons $\left(n \geqslant 2,2^{\prime}\right)$ confined in a small volume and as cluster-specific excitations. ${ }^{1}$ On the other hand, the strongest absorption bands of clusters which are due to Frenkel or intermediate-type excitons show a very complex and as-yet not well understood behavior. Even in bulk solids the nature of these excitons is still under discussion. ${ }^{2,3}$ The main difference between Frenkel and Wannier excitons is as follows. ${ }^{4}$ Wannier excitons are bound states formed from a valence-band hole and an electron in the conduction band. In this sense they are equivalent to the hydrogen atom and the radius of the exciton can be calculated according to Bohr's formula by replacing the electron and hole mass and the dielectric constant by the corresponding values in the solid. As a result, the radius of the Wannier excitons is usually much larger than the lattice constant. Wannier excitons form a series which converges toward the bottom of the conduction band and they are labeled in analogy to the hydrogen atom by the principal quantum number $n$. If the exciton radius $r_{n}$ is much smaller than the lattice constant $a$, the Wannier description fails, because the effective mass as well as the dielectric constant approximation breaks down. Here, the Frenkel-exciton model can be applied. Frenkel excitons can be regarded as excitations of single constituents of the material, which are modified by the interaction with surrounding atoms. The band structure is formed due to the resonance transfer interaction. In the special case of the 1, 1'-excitons in rare gas solids (the prime denotes the spin orbit state $j=1 / 2$ ), e.g., Ar with $r_{1}=1.9 \AA$ and $a=3.75 \AA$, the description falls in between the two regimes. For more than 30 years and still ongoing is a discussion about the correct theoretical treatment of these funda- mental excitations in rare gas solids. Different models based on the Wannier-Mott theory were applied, e.g., central cell correction $^{5,6}$ or the quantum defect model, ${ }^{7,8}$ but the results were discussed controversially. ${ }^{9}$ A key point of the controversy is whether the excitons are delocalized excitons which can move by resonant excitation transfer through the solid. More recently, the influence of the coupling between freeexciton states with local lattice vibrations ${ }^{2}$ and lattice defects $^{3}$ on optical absorption was studied theoretically using again different models.

Only very few articles have been published so far on theoretical work on the electronic structure of the tightly bound electronically excited states ( $n=1,1^{\prime}$ excitons) in rare gas clusters. The first calculations performed by Last and George $^{10}$ used a semiempirical diatomics-in-ionic systems (DIIS) method to obtain the electronic structure of $\mathrm{Ar}_{N}$ clusters $(N \leqslant 13)$. This paper gives some interesting information on the electronic properties, but the calculated width and energy range of the cluster absorption differ considerably from the experimental results. Further, the cluster size covers only the range up to $N=13$, while experimentally clusters comprising up to $10^{5}$ atoms are investigated. In this context, the energetic splitting and the intensity ratio between surface and bulk components of the first absorption bands are of special interest. According to the work of Ratner et al., ${ }^{11}$ the surface-exciton penetration depth plays an important role in the determination of electronic structure of excited states. Embedding small Ar clusters inside large Ne clusters offers the possibility of manipulating the Ar cluster surface in a well-defined way and to shed more light on the character of the strong absorbing $n=1,1^{\prime}$ excitons.

In this article, we report on fluorescence excitation spectroscopy in the vacuum-ultraviolet (VUV) spectral range of $\operatorname{Ar}_{\bar{M}}$ clusters $(\bar{M} \leqslant 100)$ embedded in large Ne clusters containing approximately 7500 atoms using a pickup technique. Strong absorption due to tightly bound excitons is observed and assigned to bulk and interface excitations. The energy shift of the absorption bands is proportional to the logarithm of the cluster size $\bar{M}$. 
The paper is organized in the following way. In Sec. II we describe the experimental setup for the investigation of doped clusters. In Sec. III the experimental results are presented. In this section we first compare the absorption of free small Ar clusters with that of embedded Ar clusters inside Ne. The second part focus on size-dependent shifts of the transition energies in doped clusters. Here, a quantitative model describing the effect is presented. In the Appendix we explain the method of the size determination of embedded clusters.

\section{EXPERIMENT}

The measurements were performed at the experimental station CLULU at the synchrotron radiation laboratory HASYLAB in Hamburg. The experimental setup is described in detail elsewhere. ${ }^{12}$ It has been modified to allow the doping of rare gas clusters with atoms from a cross-jet in a pickup process. In brief, $\mathrm{Ne}_{\bar{N}}$ clusters are prepared in a supersonic expansion through a conical nozzle $(200 \mu \mathrm{m}$ diameter, $4^{\circ}$ opening cone angle). At a nozzle temperature of $30 \mathrm{~K}$ and a stagnation pressure of 200 mbar the average cluster size was determined as $\bar{N}=7500$ using well-known scaling laws. ${ }^{13,14}$ The width [full width at half maximum (FWHM)] $\Delta N$ of the size distribution is approximately $\bar{N}$. The cluster beam intersects Ar atoms from a cross-jet on a length scale of $10 \mathrm{~mm}$. The Ar atoms are picked up by the $\mathrm{Ne}$ clusters and stick to the surface of the cluster. When several atoms are picked up the $\mathrm{Ne}$ clusters become liquid like since the collisions are warming up the cluster. Due to the increased mobility of $\mathrm{Ar}$ atoms inside the $\mathrm{Ne}$ cluster, the $\mathrm{Ar}$ atoms form small clusters inside Ne. ${ }^{15}$ A sketch of the interaction zone including the main parameters is shown in the upper part of Fig. 1. The size determination of the embedded Ar clusters is based on theoretical and experimental work published by Lewerenz et al. ${ }^{16}$ and is explained in the Appendix. According to their work, the average number $\bar{M}$ of picked-up atoms depends mainly on the Ne cluster size and the average particle density of $\mathrm{Ar}$ atoms, which is a function of the cross-jet pressure. In the middle part of Fig. 1, the Ar particle density distribution $n\left(x, y_{0}\right)$ along the beam axis $\left(x, y_{0}\right)$ is calculated for the cross-jet pressure $p_{q}$, temperature $T_{q}$, and capillary diameter $d_{q}$ of $20 \mathrm{mbar}, 300 \mathrm{~K}$, and $200 \mu \mathrm{m}$, respectively. ${ }^{17}$ The calculated average size of embedded clusters is shown in the lower part of Fig. 1. We have to point out that all numbers given below are mean values for the cluster sizes. Monochromatized synchrotron radiation $(11.5-12.9 \mathrm{eV})$ at a resolution of $2.5 \AA$ ( $\hat{=} 30 \mathrm{meV}$ bandpass) was focused $10 \mathrm{~mm}$ downstream from the nozzle on the beam. After photoexcitation the clusters emit fluorescence light, which was detected spectrally undispersed by a "solar-blind" photomultiplier (sensitivity 4-11 eV). The total VUV luminescence yield is taken as a measure of the $\mathrm{Ar}$ cluster absorption, since nonradiative decay to the ground state is inefficient in rare gas clusters. ${ }^{12}$

\section{RESULTS AND DISCUSSION}

In Fig. 2 the total VUV luminescence yield spectrum of $\mathrm{Ar}_{\overline{60}} \mathrm{Ne}_{\overline{7500}}$ clusters (b) is compared with excitation spectra of
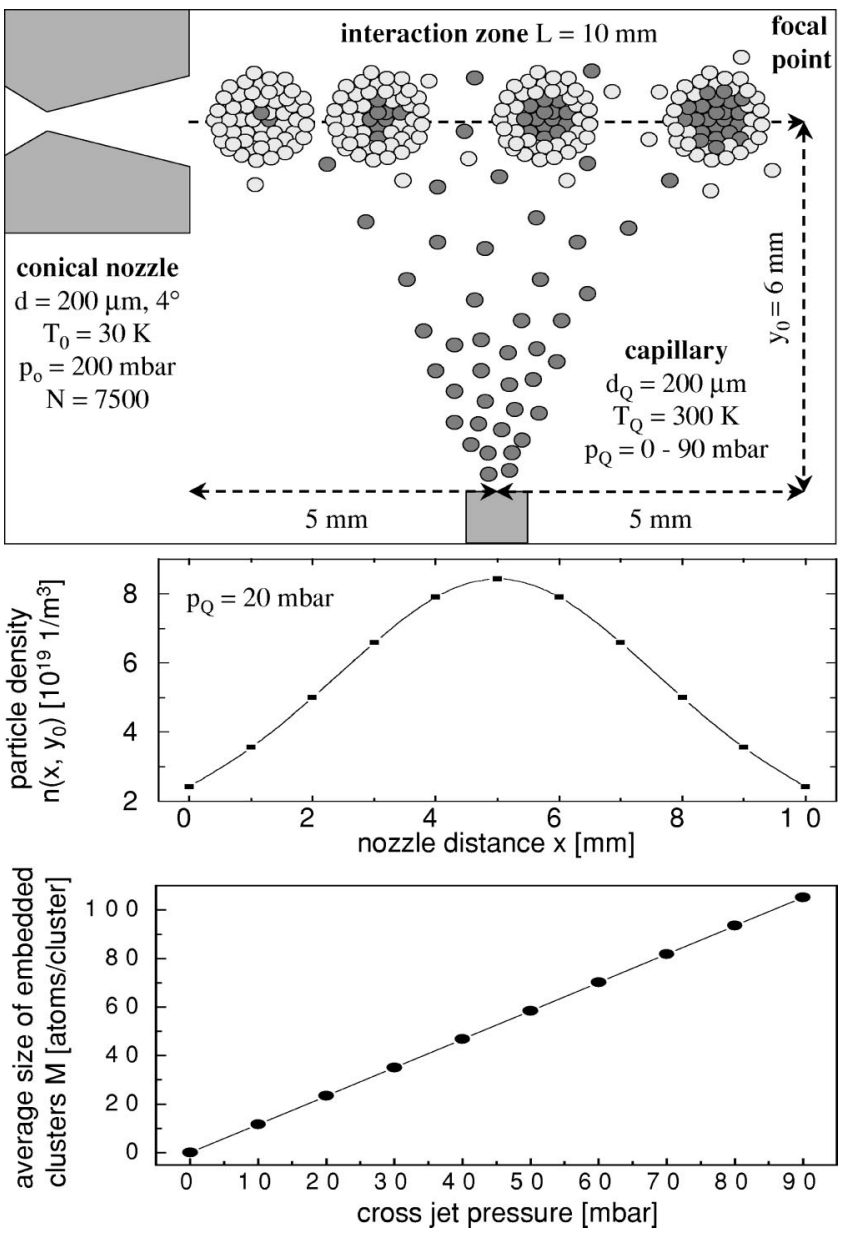

FIG. 1. Upper part: sketch of the interaction zone including the main parameters. Middle part: Ar particle density distribution along the beam axis. Lower part: calibration curve for average embedded cluster size determination.

free Ar clusters comprising $\overline{2000}$ (a) and $\overline{60}$ atoms (c), respectively. The transition energies of excitons in solid Ar are indicated in the figure. ${ }^{18}$ The intensity ratios of the different excitonic bands differ considerably from those of free clusters. In free small Ar clusters the most intense absorption bands are due to surface excitation because of the relatively high fraction of surface atoms. ${ }^{19}$ In embedded clusters these bands completely disappear, which gives strong evidence that the Ar clusters are located inside the Ne clusters. We note that surface excitons in rare gas solids are also vanishing if the surface is covered with different rare gas layers. ${ }^{20}$ In Fig. 2(b), additional absorption bands located at the highenergy side of the $1 l^{\prime}$ exciton are seen. The energy shift can be understood assuming that the excited orbital of Ar surface atoms is perturbed by the surrounding Ne cluster atoms. This absorption band at $\sim 12.65 \mathrm{eV}$ is interpreted as an excitation of the Ar-Ne interface. In small clusters it is associated with the corresponding ${ }^{1} S_{0} \rightarrow{ }^{1} P_{1}$ transition of single Ar atoms in Ne matrices ${ }^{21}$ as indicated in Fig. 2(b). By comparing Fig. 2(a) with Fig. 2(b) we can assign the other two absorption bands at $\sim 12.18 \mathrm{eV}$ and $\sim 12.41 \mathrm{eV}$ of embedded Ar clusters to the longitudinal and transverse mode of the $n=1^{\prime}$ bulk exciton. The transition energy of the $1 l^{\prime}$ exciton of 


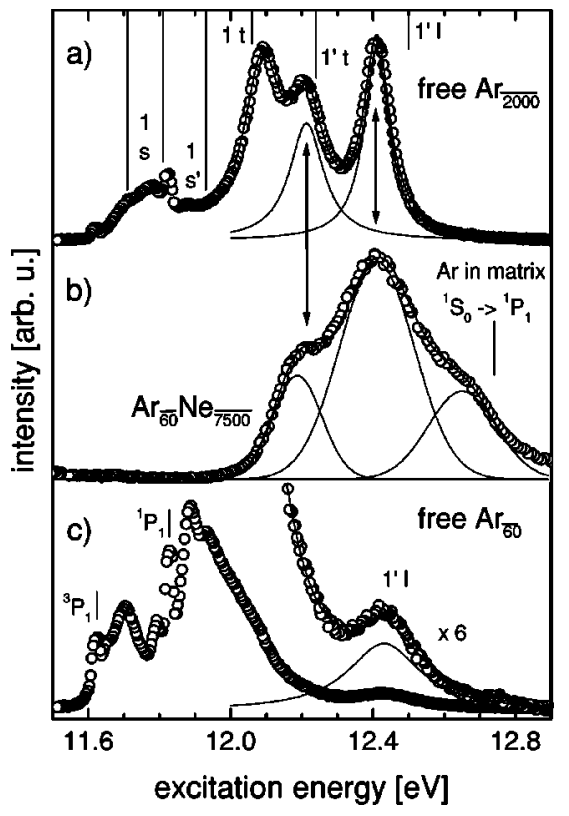

FIG. 2. VUV-fluorescence excitation spectra of (b) $\operatorname{Ar}_{\overline{60}} \mathrm{Ne}_{\overline{7500}}$ clusters and free Ar clusters containing (a) $\overline{2000}$ and (c) $\overline{60}$ atoms. Vertical lines indicate the $n=1,1^{\prime}$ excitons of solid $\mathrm{Ar}$, the atomic Ar ${ }^{1} S_{0} \rightarrow{ }^{1} P_{1}$ transition in the Ne matrix, and the ${ }^{1} S_{0} \rightarrow{ }^{1} P_{1}$ and ${ }^{1} S_{0} \rightarrow{ }^{3} P_{1}$ transition, respectively, of free Ar atoms.

embedded $\mathrm{Ar}_{\overline{60}}$ clusters is slightly shifted by approximately $\sim 20 \mathrm{meV}$ relative to the corresponding absorption energy of free $\mathrm{Ar}_{\overline{60}}$ clusters. This effect might be explained by the influence of the surrounding $\mathrm{Ne}$ atoms, since the longitudinaltransverse splitting of excitons is mainly due to the longrange dipole-dipole interaction. ${ }^{22}$ We like to note that in contrast to free small Ar clusters the $1 t$ bulk exciton is not observed in embedded clusters, which gives an indication that the oscillator strength of spin-orbit-split excitons is modified in the matrix.

The absorption bandwidths (FWHM) of excitons in rare gas clusters depend sensitively on the cluster size. ${ }^{19}$ This explains why the observed widths of the $1 t^{\prime}$ exciton $(\sim 162 \mathrm{meV})$ and the $1 l^{\prime}$ exciton $(\sim 248 \mathrm{meV})$ in small embedded $\operatorname{Ar}_{\overline{60}} \mathrm{Ne}_{\overline{7500}}$ clusters and in free $\mathrm{Ar}_{\overline{60}}$ clusters, respectively are significantly larger compared to those in free $\mathrm{Ar}_{\overline{2000}}$ clusters. Here, the absorption bandwidth of the transverse mode is $\sim 116 \mathrm{meV}$ and the width of the longitudinal mode is $\sim 87 \mathrm{meV}$.

The bulk excitations as well as the Ar-Ne interface excitation shift to lower energies as the $\operatorname{Ar}_{\bar{M}}$ cluster size increases $(40 \leqslant \bar{M} \leqslant 100)$. The size dependence of the transition energies is shown in Fig. 3. The different absorption bands are fitted with Gaussian curves. To reduce the number of free parameters the bandwidths were kept almost constant $(\bar{\sigma}$ $\approx 10 \%$ ).

In the following we present a model giving a quantitative description of the energy shift. This model has recently been used to explain the cluster size dependence of visible and near-infrared luminescence of ionic centers in photoionized Xe clusters. ${ }^{23}$ The variation of the transition energies gives direct insight into the character of the strongly bound elec-

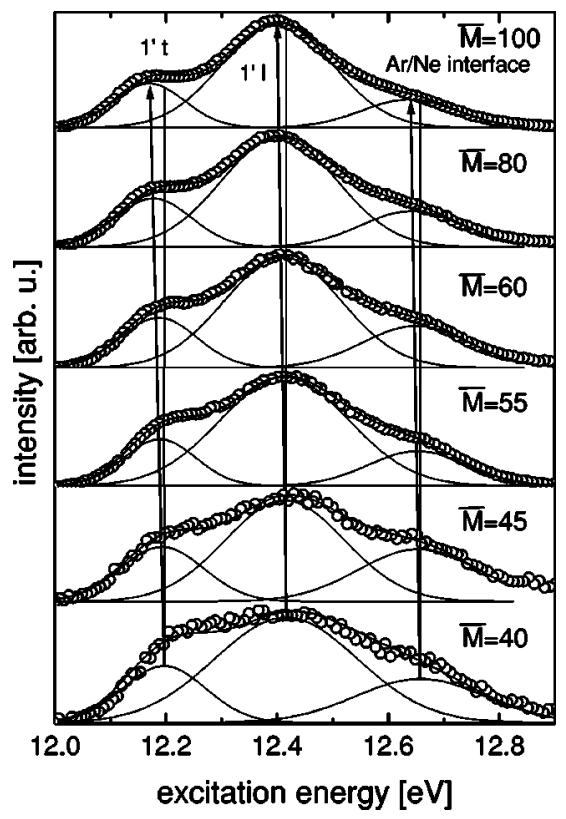

FIG. 3. VUV-fluorescence excitation spectra of $\operatorname{Ar}_{\bar{M}} \mathrm{Ne}_{\overline{7500}}$ clusters $(40 \leqslant \bar{M} \leqslant 100)$.

tronically excited states of small rare gas clusters. We like to point out that the small energy shifts of bulk and interface excitons (a few meV) could only be observed due to the suppression of the strong absorbing surface excitons by embedding the small Ar clusters inside Ne. In view of the theoretical model, the energy shift $\delta \nu$ of bulk and Ar-Ne interface excitations is plotted as a function of the logarithmic value of the average cluster size $\bar{M}$ and the number of surface atoms $\bar{M}_{S}$, respectively, in the case of the interface excitation. In Fig. 4, $\delta \nu$ is shown for different cluster sizes relative to the bulk absorption energies of $\operatorname{Ar}_{40} \mathrm{Ne}_{\overline{7500}}$ clusters and relative to the atomic $\mathrm{Ar}^{1} S_{0} \rightarrow{ }^{1} P_{1}$ transition in the $\mathrm{Ne}$ matrix at $12.74 \mathrm{eV}$ in case of the interface excitation. ${ }^{21}$ The number of surface atoms $\bar{M}_{S}$ is calculated under the assumption that the clusters have polyicosahedral structure with fivefold symmetry. ${ }^{24} \bar{M}_{S}$ is derived from a continuous curve fitted between the "magic" cluster sizes corresponding to closed-shell icosahedra. The spectral shifts can be understood within the Frenkel-exciton model. In the Frenkel model the transition energy from the ground to the excited state $(f)$ is given by ${ }^{25}$

$$
E_{f}(\vec{k})=\Delta \epsilon_{f}+D_{f}+L_{f}(\vec{k}),
$$

where $\vec{k}$ is the excitonic wave vector, $\Delta \epsilon_{f}$ is the excitation energy of the free atom, and $D_{f}$ is the environmental shift in interaction energy of one excited atom due to all surrounding atoms. The resonance interaction $L_{f}(\vec{k})$ is expressed by

$$
L_{f}(\vec{k})=\sum_{n, m=1}^{M} M_{n m}^{f} \exp [i \vec{k} \cdot(\vec{n}-\vec{m})],
$$

where $M_{n m}^{f}$ is the matrix element of the excitation transfer between atom $\vec{n}$ and $\vec{m}$. In a simple model, the electronically 

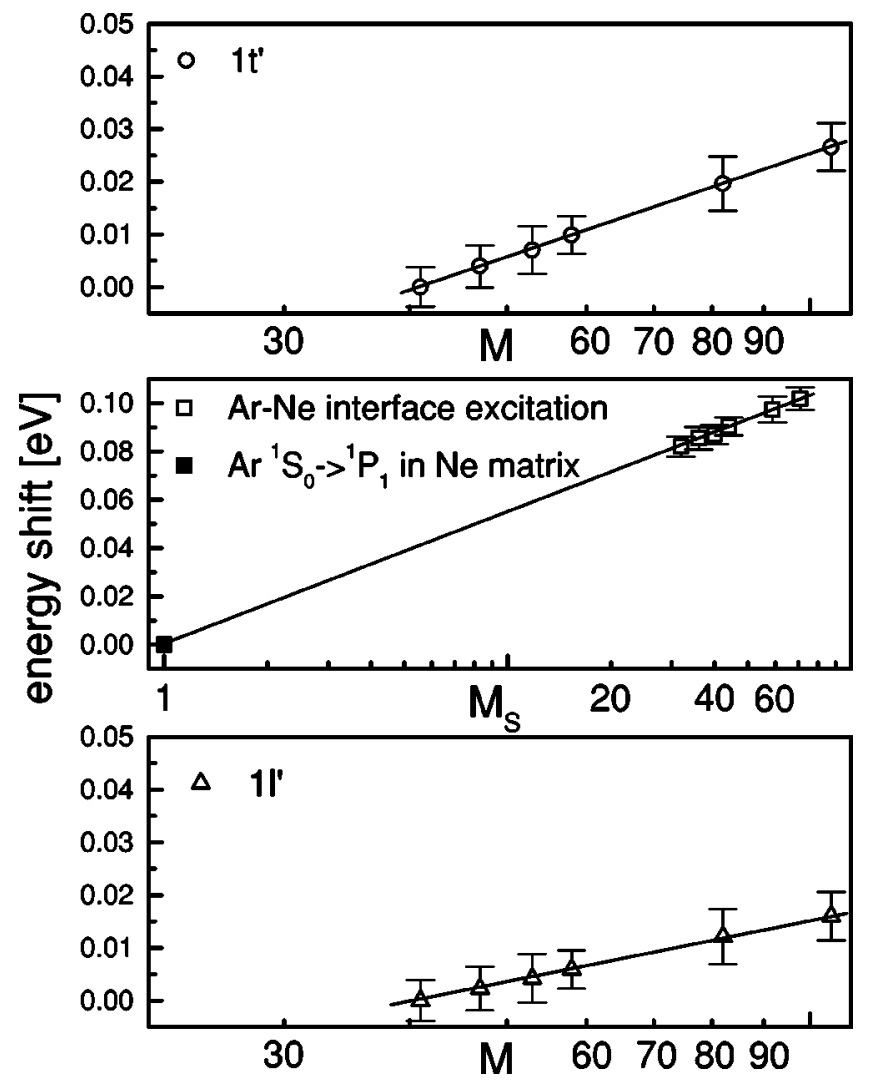

FIG. 4. Energy shift of bulk excitons (circles and triangles) and of the Ar-Ne interface excitation (cubes) as a function of the logarithmic value of, respectively, the cluster size $\bar{M}$ and the number of surface atoms, $\bar{M}_{S}$.

excited atom can be treated as a positively charged hole with an electron circulating around it. In this picture, the excitation transfer is like a "hopping" of the hole between the cluster atoms due to the dipole-dipole interaction. Since the electron movement around the positive atomic core is significantly faster than the movement of the hole, electron and hole can be treated as a combined excitation changing lattice sites due to the resonant excitation transfer. ${ }^{26}$ For the matrix element $M_{n m}^{f}$ one obtains the following expression: ${ }^{23}$

$$
M_{n m}^{f}=\frac{1}{r_{n m}^{5}}\left[\left(\vec{d}_{n} \vec{d}_{m}\right) r_{n m}^{2}-3\left(\vec{d}_{n} \vec{r}_{n m}\right)\left(\vec{d}_{m} \vec{r}_{n m}\right)\right] .
$$

Here, $\vec{r}_{n m}=\vec{n}-\vec{m}$ is the internuclear distance between different atoms $\vec{n}$ and $\vec{m}$ with transition dipole moments $\vec{d}_{n}$ and $\vec{d}_{m}$. In order to describe the cluster size dependence of the transition energy shift $\delta \nu(\bar{M})$ quantitatively we analyzed the size dependence of $D_{f}$ and $L_{f}(\vec{k})$ in Eq. (1) in detail, since for clusters it results in

$$
\delta \nu(\bar{M})=\delta D_{f}(\bar{M})+\delta L_{f}(\vec{k}, \bar{M}) .
$$

Here, $\delta D_{f}(\bar{M})$ and $\delta L_{f}(\vec{k}, \bar{M})$ are the changes in the enviromental shift and in the resonance interaction due to the increasing cluster size $\bar{M}$.
First, we focus on the environmental shift $D_{f}$ and study the effect of increasing cluster size from 40 to 100 . The above-mentioned polyicosahedral geometric structure of small rare gas clusters exhibits closed shells with increased stability for $13,55,147, \ldots$ atoms/clusters. ${ }^{27} \mathrm{Ar}_{\overline{40}}$ clusters are formed by 12 atoms around the central atom (radius of the first shell: $\left.R_{1}\right)$ and 27 atoms in the second layer $\left(R_{2}\right)$. Clusters comprising 100 atoms have closed second shells (42 atoms) and further 45 atoms in the third layer $\left(R_{3}\right)$. If we assume that the central Ar atom is excited from the ground to the excited state related to the ${ }^{1} S_{0} \rightarrow{ }^{1} P_{1}$ transition in free atoms, the key question is to what extend ground and electronically excited states are influenced by atoms from outer shells $R_{n} \geqslant R_{2}$. This question will be answered in the following.

In rare gas solids the interaction energy $D_{f}$ is calculated from the molecular potentials for $r=a$ (interatomic distances $r$ in $\operatorname{Rg}_{2}^{*}$, lattice constant of the bulk solid, $a$ ) and using the theoretical crystal potential of the polarization attraction taking the van der Waals terms into account. ${ }^{2}$ Here, we have to keep in mind that in small Ar clusters the dominant contribution to $D_{f}$ comes from the 12 atoms in the first shell, since the radius of the free atom $4 s$ orbital $\langle r\rangle=6.2 \AA$ is not much larger than the average nearest-neighbor distance $r_{0} \approx a$ $=3.75 \AA$. Last and George ${ }^{10}$ calculated the interaction energy between the excited atom and the neighboring groundstate atoms for the $\mathrm{Ar}_{13}$ cluster with the excitation at the central atom. ${ }^{10}$ According to their work, the energy of the $\mathrm{Ar}^{*}-\mathrm{Ar}_{12}$ interaction is $0.464 \mathrm{eV}$. Since both $\mathrm{Ar}_{40}$ and $\mathrm{Ar}_{100}$ clusters exhibit closed first shells, $\delta D_{f}(\bar{M})$ is mostly determined by long-range dispersion interaction from atoms of outer shells. To give an order-of-magnitude estimation for the corresponding influence on the transition energies in small Ar clusters we used the long-range potential for the excited state of $\mathrm{Ar}_{2}$, which is of the form (see, e.g., Ref. 28 or 29):

$$
V_{f}(r)=\frac{C_{3}}{r^{3}}-\frac{C_{6}}{r^{6}} .
$$

The $C_{3}$ coefficient is different from zero only if the excited state on the separated atom is dipole allowed, since it depends on the dipole matrix element $\mu_{Z}$ connecting the excited atomic state to the ground state: ${ }^{29}$

$$
C_{3} \sim\left|\mu_{Z}\right|^{2} \text {. }
$$

The $C_{6}$ van der Waals coefficient is given by the so-called "Slater-Kirkwood relation" 30

$$
C_{6}=K \frac{\alpha_{1} \alpha_{2}}{\left(\alpha_{1} / N_{1}\right)^{1 / 2}+\left(\alpha_{2} / N_{2}\right)^{1 / 2}},
$$

where $\alpha_{1}$ and $\alpha_{2}$ are the polarizabilities of the two interacting atoms, $N_{1}$ and $N_{2}$ are approximately the number of outer shell electrons, and $K$ takes the value of 15.7 to obtain $C_{6}$ in $\left[\mathrm{eV} \AA^{6}\right] .{ }^{31}$ The polarizability of the ground-state argon atom is well known, ${ }^{31}$ whereas experimental and theoretical data on polarizabilities of electronically excited atoms are very 


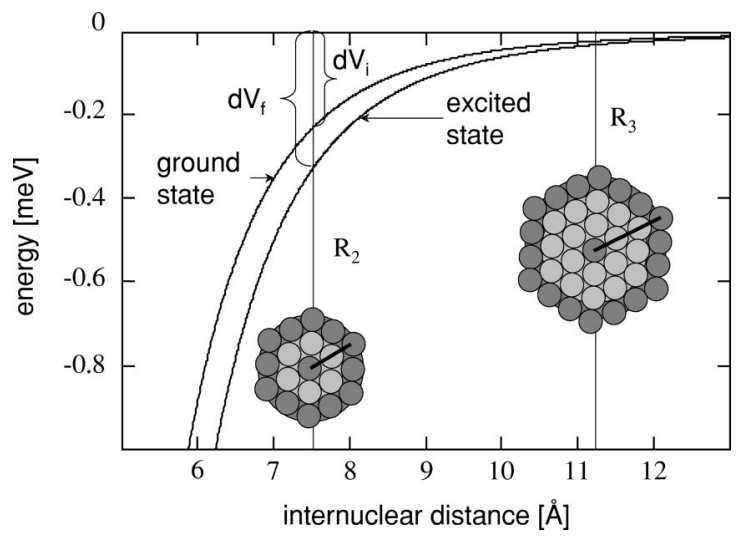

FIG. 5. $-C_{6} / r^{6}$ contribution to the long-range van der Waals potential of ground $(i)$ and excited $(f) \mathrm{Ar}_{2}$ states relative to the atomic ${ }^{1} S_{0}$ and ${ }^{1} P_{1}$ dissociation limit as a function of the internuclear distance. The central cluster atom is excited, $R_{2}$ and $R_{3}$ denote the radii of the second and third icosahedral shells, and $d V_{i, f}$ describe the gain in polarization energy due to each atom in the respective layer.

rare. Since $C_{6}$ is approximately proportional to the atoms volume, ${ }^{10}$ we estimate its value from theoretical work on excited molecular states of $\mathrm{Ne}_{2}$ published by Cohen and Schneider. ${ }^{29}$ We obtain $C_{6} \approx 58.3 \mathrm{eV}^{6}$ for Ar dimers in electronically excited molecular states correlated with the atomic ${ }^{1} P_{1}$ energy level.

In Fig. 5, we plotted the attractive $-C_{6} / r^{6}$ term of both ground- and excited-state potentials of $\mathrm{Ar}_{2}$ relative to the asymptotic atomic ${ }^{1} S_{0}$ and ${ }^{1} P_{1}$ states, respectively, as a function of the internuclear distance $r$. The $C_{3} / r^{3}$ term is the molecular analog of the resonant excitation transfer $L_{f}(\vec{k})$ in bulk solids and will be discussed later. It is due to the delocalization of the excited electron. In order to make sure that we do not count the effect of delocalization and hopping of the excitation twice, we have to set $C_{6}$ to zero if the term $\delta L_{f}(\vec{k}, \bar{M})$ is considered.

To estimate the change of the transition energy with cluster size due to long-range dispersion forces from outer-shell atoms, we assume that the central atom of the cluster is excited. In this picture the change of the interaction energy $\delta D_{f}(\bar{M})$ can be approximated by the sum of the contributions of different atoms $d V_{i, f}$ in outer shells. The polarization energy results in a decrease of both ground state $(i)$ and excited state $(f)$. The second and third icosahedral shell radii of rare gas clusters $\left(R_{2}, R_{3}\right)$ are approximately 2 and 3 times, respectively, the average nearest-neighbor distance $r_{0}: R_{2}$ $\approx 7.5 \AA$ and $R_{3} \approx 11.25 \AA$. For the excited state of $\operatorname{Ar}_{100}$ clusters, we obtain a decrease of the potential energy of about $6.21 \mathrm{meV}$ relative to that of $\mathrm{Ar}_{\overline{40}}$ clusters. In case of the ground-state potential, the energy is decreased by 4.36 $\mathrm{meV}$.

In conclusion, taking only the van der Waals polarization into account, the transition energy of Ar clusters containing 100 atoms is lowered by approximately $1.85 \mathrm{meV}$ with respect to clusters containing 40 atoms. This value is more than one order of magnitude smaller compared with the experimentally observed energy shift of $\sim 20 \mathrm{meV}$. The cluster size dependence of the interaction energy $\delta D_{f}(\bar{M})$ is not the dominant effect, although as we have mentioned above, the absolute value of $D_{f}$ for a given cluster size is relatively large [e.g., $\operatorname{Ar}_{13}: D_{f}=464 \mathrm{meV}$ (Ref. 10)].

Therefore, $\delta \nu(\bar{M})$ is mainly determined by the resonance interaction term $L_{f}(\bar{M})$. In the following we analyze its cluster size dependence in detail. Because the radius $R_{c l}$ of the embedded $\operatorname{Ar}_{\bar{M}}$ clusters $(\bar{M} \leqslant 100)$ is small compared to the wavelength $\lambda$ of the excitation radiation $\left(R_{c l} \leqslant 1 \mathrm{~nm}\right)$, one can replace the sum in Eq. (2) by an integration of the different contributions of $M_{n m}^{f}$. One obtains ${ }^{23}$

$$
\delta \nu(\bar{M})=-\alpha \frac{d^{2}}{3 \epsilon r_{0}^{3}} \ln (\bar{M}) .
$$

Here, $\alpha=4.14$ is a numerical constant, $d$ is the strength of the transition dipole moment $\left(d_{n}=d_{m}=d / \sqrt{\epsilon}\right), r_{0}$ is the nearest-neighbor distance, and $\epsilon$ is the dielectric constant. The theoretically predicted linear dependence $\delta \nu \sim \ln (\bar{M})$ is in very good agreement with the experimental data points in Fig. 4. This gives strong evidence that the electronically excited strongly bound first excited states in small Ar clusters can be described in the Frenkel model taking the sizedependent resonant excitation transfer into account. A description in the quantum defect model is not possible, since here the excitation transfer is not explicitly considered. On the other hand, one has to point out that our approximation is only valid in the molecular limit $\left(R_{C l} \ll \lambda\right)$ where the energetic positions of the bulk excitons in clusters do not converge toward the bulk solid limit. This is somehow surprising since other energetic size effects, e.g., spectral shifts of large organic molecules embedded in rare gas clusters $\left(R g_{N}\right)$ converge toward their bulk values in the molecular limit, ${ }^{32}$ while electrodynamic size effects, e.g., radiative lifetimes, converge toward their bulk values only in the so-called electrodynamic regime $\left(R_{C l} \geqslant \lambda\right){ }^{33}$ Interestingly, in the molecular limit $(N \leqslant 1000)$, the lifetime modification $\Delta \tau_{r}$ of embedded aromatic molecules depends on the cluster size and can be approximated by $\Delta \tau_{r} / \tau_{r}^{0} \sim \ln (N)$, where the lifetime $\tau_{r}^{0}$ is that of the free molecule. ${ }^{34}$

\section{CONCLUSION}

We have recorded VUV-fluorescence excitation spectra of small embedded $\operatorname{Ar}_{\bar{M}}$ clusters $(\bar{M} \leqslant 100)$ inside large $\mathrm{Ne}_{\overline{7500}}$ clusters. The absorption bands in the energy range 11.5-12.9 $\mathrm{eV}$ were assigned to the longitudinal and transverse modes of the Ar $1^{\prime}$ exciton and to an excitation of the interface between the embedded Ar cluster and the Ne host cluster. The observed energy shift with cluster size is proportional to the logarithm of the cluster size $\bar{M}$ and the number of surface atoms $\bar{M}_{S}$, resectively, in case of the Ar-Ne interface excitation. This is in agreement with the Frenkel-exciton model and points out that the resonant excitation transfer plays an important role and has to be considered in theoretical models describing intermediate excitons in rare gas clusters and the bulk solid. 


\section{ACKNOWLEDGMENTS}

We thank Prof. G. Zimmerer (University of Hamburg) for very helpful discussions. The financial support for this work was provided by the Deutsche Forschungsgemeinschaft under Grant No. Mo719/1-3 and is gratefully acknowledged.

\section{APPENDIX}

The probability $P$ for a Ne cluster (capture cross section $\sigma_{\text {cap }}$ ) passing through the interaction zone (length $L$ and average particle density $\bar{n}_{S}$ ) and picking up $k$ atoms is described by Poisson statistics: ${ }^{16}$

$$
P_{k}(L)=\frac{\left(\sigma_{c a p} \bar{n}_{S} L\right)^{k}}{k !} \exp \left[-\sigma_{c a p} \bar{n}_{S} L\right] .
$$

The cross-jet particle density along the cluster beam axis $\left(x, y_{0}\right)$ for cross-jet pressures $p_{Q}$, temperatures $T_{Q}$, and capillary diameters $d_{Q}$ is given by ${ }^{17}$

$$
n\left(x, y_{0}\right)=C_{1} \cos ^{2}\left(C_{2}\right) \cos ^{2}\left[\frac{\pi}{2 \Phi} C_{2}\right],
$$

with

$$
C_{1}=\frac{Z_{P} d_{Q}^{2} p_{Q}}{y_{0}^{2} k_{B} T_{Q}} \text { and } C_{2}=\arctan \left(\frac{x-L / 2}{y_{0}}\right) .
$$

In this equation $Z_{P}=0.157$ and $\Phi=1.365$ are numerical constants for rare gases ${ }^{17}$ and $k_{B}$ is the Boltzmann constant. The average particle density $\bar{n}_{S}$ is simply

$$
\bar{n}_{S}=\frac{1}{L} \int_{0}^{L} n\left(x, y_{0}\right) d x \text {. }
$$

In a crude approximation, we assume that the sum of the $\mathrm{Ne}_{\bar{N}}$ cluster cross section and the atomic cross section is equal to the capture cross section. Monte Carlo simulations and experiments on the scattering and capture cross section of $\mathrm{He}$ clusters show that the effective geometric cross section $\sigma_{\text {geo }}$ for light atoms (e.g., Ar) or large $\mathrm{He}_{\bar{N}}$ clusters $(\bar{N}>3000)$ is in good agreement with the measured capture cross section $\sigma_{\text {cap }} \cdot{ }^{16,35}$ In the case of Ar-doped $\mathrm{He}_{\overline{3000}}$ clusters Lewerenz et al. obtained for the so-called "sticking coefficient" a value of $s=\sigma_{\text {cap }} / \sigma_{\text {geo }} \approx 0.94$. $^{16,35}$ In this approximation we get

$$
\begin{gathered}
\sigma_{c a p} \approx \sigma_{g e o}=\pi\left(R_{C l}+r\right)^{2}, \\
\sigma_{c a p} \approx \pi\left[\left(\frac{3}{4 \pi \rho} N\right)^{1 / 3}+r\right]^{2} .
\end{gathered}
$$

Here, $R_{c l}$ is the cluster radius, $r$ is the radius of the embedded atom, and $\rho$ is the particle density in Ne solids.

While small clusters are formed inside large Ne clusters, $\mathrm{Ne}$ atoms are evaporated from the Ne cluster surface (cooling mechanism) and the cluster cross section decreases, respectively, the capture cross section. In a simple model the deposited condensation energy inside Ne clusters as well as the energy due to the evaporation of $\mathrm{Ne}$ atoms is proportional to the binding energy per atom, $E_{b}$, of the respective solid. In this picture, $\mathrm{Ne}_{\overline{7500}}$ clusters doped with $100 \mathrm{Ar}$ atoms evaporate approximately $335 \mathrm{Ne}$ atoms $\left[E_{b}(\mathrm{Kr}) / E_{b}(\mathrm{Ne})\right.$ $\approx 3.35]$ and the cluster cross section is therefore reduced by roughly $3 \%$. Since this is a rather small effect, we treat the capture cross section as constant and calculate the average size $\bar{M}$ of the embedded Ar cluster as a function of the cross jet pressure $p_{Q}$ using Eqs. (A1)-(A5):

$$
\bar{M}\left(p_{Q}\right)=\int_{1}^{\infty} k P_{k}(L) d k .
$$

*Electronic address: tim.laarmann@desy.de

†Present address: Ruhr-Universität-Bochum, 4478 Bochum, Germany.

${ }^{1}$ J. Wörmer, R. Karnbach, M. Joppien, and T. Möller, J. Chem. Phys. 104, 8269 (1996).

${ }^{2}$ I. Fugol, Low Temp. Phys. 25, 713 (1999).

${ }^{3}$ A.M. Ratner, Phys. Lett. A 269, 245 (2000).

${ }^{4}$ N. Schwentner, E. E. Koch, and J. Jortner, Electronic Excitations in Condensed Rare Gases (Springer-Verlag, Berlin, 1985).

${ }^{5}$ J. Hermanson and J.C. Phillips, Phys. Rev. 150, 652 (1966).

${ }^{6}$ J. Hermanson and J.C. Phillips, Phys. Rev. 150, 660 (1966).

${ }^{7}$ L. Resca, R. Resta, and S. Rodriguez, Phys. Rev. B 18, 696 (1978).

${ }^{8}$ L. Resca, R. Resta, and S. Rodriguez, Phys. Rev. B 18, 702 (1978).

${ }^{9}$ V. Saile, R. Reininger, P. Laporte, I.T. Steinberger, and G.L. Findley, Phys. Rev. B 37, 10901 (1988).

${ }^{10}$ I. Last and T.F. George, J. Chem. Phys. 98, 6406 (1993).

${ }^{11}$ M.A. Ratner, E.T. Verkhovtseva, and A.M. Ratner, J. Lumin. 68, 255 (1996)

${ }^{12}$ R. Karnbach, M. Joppien, J. Stapelfeld, J. Wörmer, and T. Möller, Rev. Sci. Instrum. 64, 2838 (1993).

${ }^{13}$ O.F. Hagena, Z. Phys. D: At., Mol. Clusters 4, 291 (1987).
${ }^{14}$ U. Buck and R. Krohne, J. Chem. Phys. 105, 5408 (1996).

${ }^{15}$ S. Goyal, D.L. Schutt, and G. Scoles, J. Chem. Phys. 102, 2302 (1995).

${ }^{16}$ M. Lewerenz, B. Schilling, and J.P. Toennies, J. Chem. Phys. 102, 8191 (1995).

${ }^{17}$ D. R. Miller, Atomic and Molecular Beam Methods (Oxford University Press, Oxford, 1988), Vol. 1.

${ }^{18} \mathrm{G}$. Zimmerer, in Creation, Motion and Decay of Excitons in RareGas Solids, edited by U. M. Grassano and N. Terzi (Soc. Italiana di Fisca, Bologna, 1987).

${ }^{19}$ J. Wörmer, M. Joppien, G. Zimmerer, and T. Möller, Phys. Rev. Lett. 67, 2053 (1991).

${ }^{20}$ V. Saile, M. Skibowski, W. Steinmann, P. Gürtler, E.E. Koch, and A. Kozevnikov, Phys. Rev. Lett. 37, 305 (1976).

${ }^{21}$ N. Schwentner, Appl. Opt. 19, 4104 (1980).

${ }^{22}$ V. Chandrasekharan and E. Boursey, Phys. Rev. B 19, 3299 (1979).

${ }^{23}$ A.V. Kanaev, M.C. Castex, L. Museur, R. von Pietrowski, and T. Möller, Phys. Rev. Lett. 75, 2674 (1995).

${ }^{24}$ S. Kakar, O. Björneholm, J. Weigelt, A.R.B. de Castro, L. Tröger, R. Frahm, T. Möller, A. Knop, and E. Rühl, Phys. Rev. Lett. 78, 1675 (1997).

${ }^{25}$ A. S. Davidov, Theory of Molecular Exzitons (Plenum, New York, 1971). 
${ }^{26}$ I. Last and T.F. George, Chem. Phys. Lett. 183, 547 (1991).

${ }^{27}$ M.R. Hoare, Adv. Chem. Phys. 40, 49 (1979).

${ }^{28}$ F.X. Gadea, F. Spiegelmann, M.C. Castex, and M. Morlais, J. Chem. Phys. 78, 7270 (1983).

${ }^{29}$ J.S. Cohen and B. Schneider, J. Chem. Phys. 61, 3230 (1974).

${ }^{30}$ J. C. Slater and J.G. Kirkwood, Phys. Rev. 37, 682 (1931).

${ }^{31}$ R. Cambi, D. Cappelletti, G. Liuti, and F. Pirani, J. Chem. Phys. 95, 1852 (1991).
${ }^{32}$ E. Shalev, N. Ben-Horin, U. Even, and J. Jortner, J. Chem. Phys. 95, 3147 (1991).

${ }^{33}$ A. Penner, A. Amirav, J. Jortner, and A. Nitzan, J. Chem. Phys. 93, 147 (1990).

${ }^{34}$ J. Jortner, Z. Phys. D: At., Mol. Clusters 24, 247 (1992).

${ }^{35}$ M. Lewerenz, B. Schilling, and J.P. Toennies, J. Chem. Phys. 106, 5787 (1997). 